\title{
LA RISPECIFICAZIONE DEL TRASMETTITORE E DEL RECETTORE È UNA NUOVA FORMA DI PLASTICITÀ DEL SISTEMA NERVOSO NELL'ADULTO
}

\author{
FRANCESCO CLEMENTI (*)
}

\begin{abstract}
SunTo. - Dopo alcune parole sul ruolo scientifico del prof Paolo Mantegazza all'Università di Milano $(4,5,8)$, illustro brevemente alcuni studi sulla rispecificazione trasmettitoriale e recettoriale nell'animale adulto in seguito a particolari situazioni sperimentali. Le eccezionali scoperte del primo novecento sulla comunicazione neuronale hanno permesso di stabilire che la maggioranza delle comunicazione tra cellule nervose avviene tramite una speciale struttura, la sinapsi, che permette il passaggio unidirezionale dell'informazione tra due cellule attraverso la liberazione di un neurotrasmettitore dalla cellula presinaptica e il suo riconoscimento dalla cellula postsinaptica. Secondo l'assioma di $\mathrm{H}$. Dale ogni neurone poteva essere identificato in base al neurotrasmettitore che sintetizzava e rilasciava e la cellula innervata dal tipo di recettori che esprimeva; quindi un neurone era eccitatorio se liberava acetilcolina, glutammato o altri trasmettitori, o inibitorio se liberava GABA o glicina (9). Studi ulteriori dimostrarono che, soprattutto durante lo sviluppo, un neurone può rilasciare e co-rilasciare trasmettitori diversi, talvolta anche contemporaneamente dimostrando una grande plasticità dinamica aprendo un nuovo campo di studio sulla plasticità sinaptica: la rispecificazione del trasmettitore e dei recettori. Il nostro gruppo ha contribuito al suo studio cercando di "forzarla" attraverso esperimenti di de-nervazione e re-innervazione eterologa dei gangli del sistema nervoso autonomo e della muscolatura liscia e striata. All'inizio abbiamo studiato le capacità rigenerative del sistema nervoso periferico in tre modelli sperimentali: a) re-innervazione del ganglio cervicale superiore (SCG) denervato da parte di fibre colinergiche efferenti vagali, b) re-innervazione di muscoli effettori periferici (membrana nittitante) da parte di fibre colinergiche pregangliari; c) innervazione in un modello di trapianto in vivo di organi periferici da parte del SCG $(14,15,22)$. In queste ricerche abbiamo stabilito: 1) che un ganglio simpatico poteva essere reinnervato da fibre provenienti dal sistema vagale ottenendo una funzionalità normale della sinapsi gangliare,
\end{abstract}

(*) Istituto Lombardo Accademia di Scienze e Lettere, Università degli Studi di Milano, Italia. E-mail: francesco.clementi@unimi.it 
ma con una ristrutturazione dei circuiti nervosi centrali in quanto, in vivo, stimoli fisiologici di natura simpatica, avvenivano attraverso una eccitazione vagale e non simpatica; 2) che fibre pregangliari colinergiche potevano innervare direttamente la muscolatura liscia adrenergica rilasciando catecolamine invece di acetilcolina; 3) che in un sistema artificiale di trapianto in vitro di SCG assieme a iride o midollare del surrene, il SCG era capace di distinguere tra organi che richiedevano una innervazione postsinaptica, iride, che veniva innervata, da organi che richiedono una innervazione presinaptica, midollare del surrene che non veniva innervata. Questi risultati dimostrano la grande plasticità di risposta del sistema nervoso periferico adulto in vivo a situazioni non fisiologiche che riesce a modificare le proprietà biologiche di neuroni, sconvolge e riconverte circuiti neuronali centrali, ma continua a mantenere regole fondamentali tra neuroni innervanti e cellule innervate in situazioni di trapianti sperimentali isolati dai circuiti nervosi. Trenta anni dopo, il gruppo del prof. Brunelli di Brescia (23), assieme a farmacologi e fisiologi, aveva evidenziato la possibilità di re-innervare in modo funzionale muscoli striati denervati con fibre nervose che derivavano dal nucleo rosso e dalla formazione reticolare del complesso vestibolare. L'interesse consisteva, ancora una volta, nel fatto che le fibre innervanti erano di tipo glutammatergico, e non colinergiche come quelle dei normali motoneuroni, e che la trasmissione neuromuscolare si tramutava da colinergica nicotinica in glutammatergica. Un nuovo tipo di plasticità: la rispecificazione recettoriale (25). In stretta collaborazione tra il nostro gruppo milanese e il gruppo bresciano abbiamo potuto riconfermare che la re-innervazione avvenuta nella nuova placca neuromuscolare era di natura glutamatergica; che le nuove terminazioni reinnervavano le fibre muscolari negli stessi siti prima innervati dalle fibre colinergiche; che le nuove fibre re-innervanti liberavano glutammato; e che le cellule muscolari esprimevano ex novo recettori per il glutammato. Ancora una volta eravamo in presenza di un fenomeno straordinario di plasticità sinaptica, in questo caso una rispecificazione recettoriale, e sempre accompagnato da forti ripercussioni sui circuiti del sistema nervoso centrale. Queste esperienze, assieme a molte altre ormai presenti in letteratura, dimostrano che il sistema nervoso periferico adulto, sia autonomo che muscoloscheletrico, ha una plasticità prima impensabile e aprono un campo di grande interesse biologico per capire come venga regolata la specificità neuronale e per immaginare esperimenti di re-innervazione non canonici ma forse ugualmente funzionali in situazioni di trapianto e di denervazione traumatica.

$$
* * *
$$

AbstRact. - After some words on the scientific role of Professor Paolo Mantegazza at the University of Milan $(4,5,6)$, I briefly illustrate some studies related to the occurrence of neurotransmitter and receptor re-specification in the adult animals. The great discoveries of the early twentieth century on neuronal communication have established that the majority of communication between nerve cells occurs through a special structure, the synapse, allowing the one-way transfer of information between two cells through the release of a neurotransmitter from the presynaptic cell and its recognition by receptors localized in the postsynaptic cell. According to H. Dale axiom (9) each neuron could be identified on the basis of the neurotransmitter released and the innervated cell by the type of receptors expressed; then neurons could be classified as excitatory if they release acetylcholine, glutamate or other transmitters, or inhibitory if they release GABA or glycine. However, in recent years many studies have shown that, 
especially during development, a neuron could release and co-release several neurotransmitters, sometimes even simultaneously, changing its classification from excitatory to inhibitory and vice versa (7). This researches opened a new field of study on synaptic plasticity: the neurotransmitter and receptor re-specification. Our group, together with Prof. Mantegazza, tried to "force" it through experiments of denervation and heterologous re-innervation in the autonomic nervous system and at the neuromuscular junction. In a first series of experiments we studied the regenerative capabilities of the peripheral nervous system in three experimental models: a) re-innervation of the denervated superior cervical ganglion (SCG) $(14,15,22)$ by cholinergic efferent vagal fibers, b) re-innervation of peripheral effectors smooth muscles (nictitating membrane) by the cholinergic preganglionic fibers; c) re-innervation in an in vivo transplant model of peripheral organs by the SCG. In these researches we have established: 1) that a sympathetic ganglion could be re-innervated by vagal fibers forming normal ganglionic synapses, but with a strong reshaping, in vivo, of the central neural circuits so that sympathetic stimuli occurred through a vagal excitation; 2) preganglionic cholinergic fibers innervate the smooth muscle of the nictitating membrane releasing catecholamines instead of acetylcholine; 3) that in an in vivo model of SCG transplant together with iris or adrenal medulla fragments, the SCG was able to distinguish between organs that required a postsynaptic innervation, iris, which was innervated, and organs that require a presynaptic innervation, the adrenal medulla, that was not innervated. We were then in the presence, even in the adult animal, of a new nervous plasticity with re-specification the neurotransmitter. These results demonstrate that heterologous innervation could "force" plasticity in adult peripheral nervous system, alters the biological properties of neurons, upsets central neuronal circuits, but continues to maintain in experimental transplants basic rules of innervation between neurons and peripheral organs. Thirty years later, the group of prof. Brunelli in Brescia (23), along with pharmacologists and physiologists, had highlighted the possibility of re-innervate striated muscles in a functional way with nerve fibers derived from the red nucleus of the vestibular complex. The interest was, once again, in the fact that the re-innervating fibers were of glutamatergic type, and not cholinergic like those of normal motor neurons, and that neuromuscular transmission was transformed from nicotinic cholinergic in glutamatergic. A new type of plasticity: the receptor respecification had occurred also in this experimenal model. In close cooperation between our Milan and the Brescia group we could reconfirm with more appropriate experiments that the re-innervation occurred; that neuromuscular junction had a glutamatergic transmission; that new re-innervating fibers made synapses at the same sites of the previous neuromuscular junctions; that the new fibers release glutamate; and that muscle cells expressed new glutamate receptors (24). Once again we were in the presence of an extraordinary phenomenon of synaptic plasticity, in this case a receptor re-specification, and again with a strong impact on the central nervous system circuits. These experiences, along with many others now available in the literature, show that the adult peripheral nervous system, both autonomous and musculoskeletal, has a plasticity unthinkable before and open a field of great interest aiming at the understanding how neuronal specificity is regulated and at the investigation of non-canonical, but perhaps functional, re-innervation experiments in transplants and in posttraumatic surgery. 


\section{INTRODUZIONE}

Prima di illustrare questo specifico argomento di ricerca, svolto in gran parte con il prof. Mantegazza, direttamente o indirettamente attraverso i suoi continui consigli e discussioni, vorrei richiamare brevemente l'atmosfera scientifica e culturale che permeava l'allora Istituto di Farmacologia dell'Università di Milano, poi divenuto Dipartimento di Farmacologia "Emilio Trabucchi" e recentemente confluito nel Dipartimento di Biotecnologie Mediche Traslazionali. Non mi soffermerò sulle molte e positive attività svolte dal prof. Mantegazza in favore dell'Università in tutti i ruoli che egli ha ricoperto nella sua lunga vita accademica perché saranno molto meglio illustrate nella seconda parte di questa giornata. Vorrei solo accennare che esse hanno, probabilmente, origine nella lunga frequentazione e discussione con il Prof. Emilio Trabucchi, direttore e fondatore dell'Istituto di Farmacologia, che aveva ben saputo amalgamare l'attività scientifica di alto livello svolta in Istituto con quella più generale "politica" che vedeva l'attività scientifica e culturale non solo come esaltante esperienza intellettuale ma anche, e soprattutto, come elemento essenziale di sviluppo culturale ed economico del Paese che allora si stava formando dopo la stasi drammatica della seconda guerra mondiale. La ricerca non aveva senso se rimaneva isolata dal contesto sociale del Paese. Il Paese non poteva, e non può, permettersi che essa si inaridisca e non riesca a svolgere al meglio le sue funzioni di guida e di propulsione. Per il prof. Trabucchi questo valeva a maggior ragione per la farmacologia che ha lo scopo di migliorare la vita delle persone singole e della comunità.

In questa atmosfera di rinnovamento culturale e di entusiasmo rinasceva la ricerca scientifica in Istituto, pur con la ristrettezza dei mezzi e con le tecnologie che oggi riterremo un po' primitive, alle quali suppliva l'inventiva dei ricercatori, l'intensa discussione corale sugli esperimenti e il continuo ricorso alla biblioteca che da subito fu resa internazionale ed aggiornata con le riviste più rinomate nel settore della fisiologia e farmacologia, ma anche della patologia e della clinica in una accezione assai ampia della scienza biomedica.

Per questi motivi e per la personalità eccezionale del suo direttore, il prof Emilio Trabucchi, che sosteneva questa moderna impostazione della ricerca universitaria senza gelosie, ma semmai sempre cercando lo stimolo più opportuno per ciascuno perché migliorasse la sua atti- 
vità, l'Istituto fu subito una calamita che ha richiamato tra le sue mura non solo ricercatori di base, ma anche clinici e patologi, non solo italiani ma anche stranieri (1).

In questa comunità dalla formazione così diversa ma unita nell'avventura scientifica (ben raffigurata nella Fig. 1) si veniva a creare una ricerca che, prendendo spunto dalla patologia e da quegli aspetti funzionali ancora ignoti, cercava di metterne in luce i meccanismi con i quali si effettuavano e la possibilità di modularli attraverso i farmaci. La farmacologia assumeva quindi una visione complessiva molto ampia che gli permetteva di fare da giunzione tra la patologia e la fisiologia cercando di migliorare le prospettive terapeutiche allora ancora molto scarse.

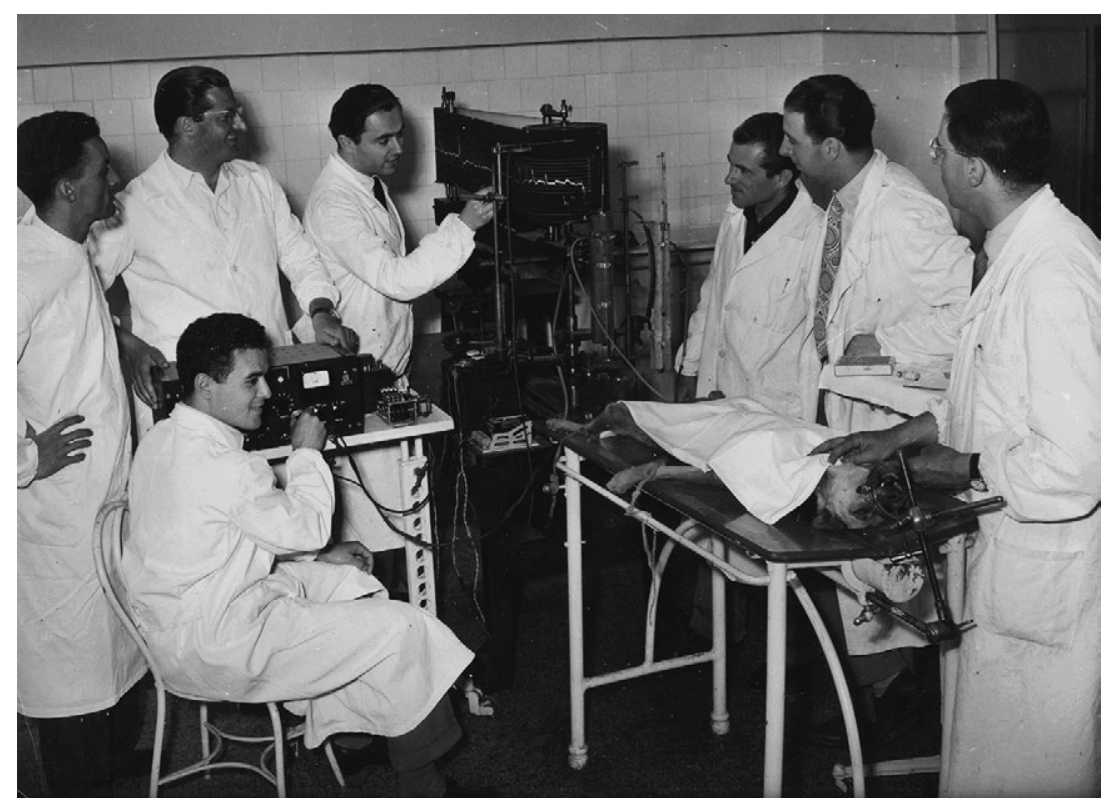

Fig. 1. Gruppo di ricercatori che sta conducendo, all'Istituto di Farmacologia all'inizio degli anni 1960, un esperimento in vivo registrando al chimografo gli effetti di farmaci sulla pressione arteriosa, sul respiro e su alcune funzioni del sistema simpatico. Sono presenti ricercatori che negli anni hanno poi raggiunto posizioni di prestigio nel campo della chirurgia generale, della chirurgia plastica, della clinica medica, della otorinolaringoiatria e della farmacologia. E' da notare la natura corale della conduzione dell'esperimento e della discussione dei dati che man mano emergevano. E' interessante sottolineare che l'attività sperimentale veniva eseguita sempre dopo le lunghe giornate di lavoro in clinica e che spesso le sedute si chiudevano a notte inoltrata. 
In questo ambiente il prof. Mantegazza si inserisce fin da studente, ne matura le sue convinzioni e motivazioni personali che lo hanno portato a svolgere un ruolo così importante e complesso nello sviluppo della ricerca e dell'Università nella società che manterrà fino alla fine della sua carriera e ben oltre la fine burocratica della carriera accademica. Certamente peculiare della figura di Mantegazza è il fatto di aver sempre coltivato e mai oscurato il suo profondo ed originale interesse scientifico anche nei momenti più intensi della sua vita di Amministratore dell'Università. Personalmente ricordo la sua grande disponibilità nei miei confronti alle prime armi nella ricerca scientifica sulle culture cellulari, la trasferta fatta assieme a Cambridge per accompagnarmi alla mia prima esperienza internazionale di lavoro, le belle e stimolanti e sempre positive discussioni sui progetti di ricerca e sull'Università che abbiamo avuto fino all'ultimo, anche partendo talvolta da punti di vista molto diversi, e il continuo supporto al nostro gruppo di ricerca come ben testimoniano le due foto che lo ritraggono mentre introduce in un nostro simposio nel 1996 i proff. George Palade e Paul Greengard, premi Nobel, ricercatori che molto hanno dato allo sviluppo della biologia cellulare e molecolare $(2,3)$ e "numi tutelari" del nostro gruppo fin dalla sua nascita (Fig. 2).

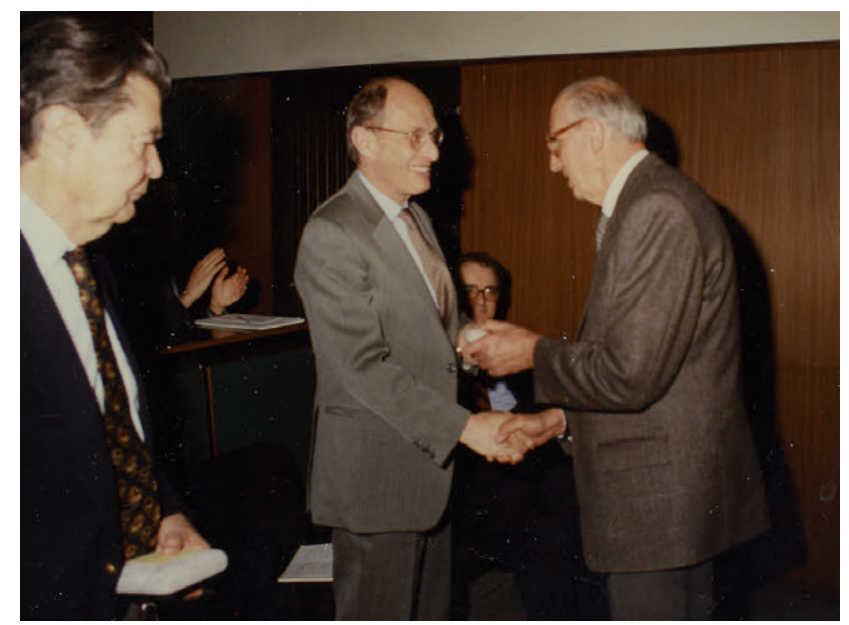

Fig. 2. Il prof. Paolo Mantegazza, Rettore dell'Università di Milano, consegna le medaglie d'oro del Dipartimento di Farmacologia e della Fondazione Trabucchi al prof. George Palade e al prof. Paul Greengard durante il simposio svoltosi in Dipartimento nel 1996 per $i 25$ anni di attività del Centro CNR di Farmacologia Cellulare e Molecolare. 


\section{LA PLASTICITÀ SINAPTICA E LA RISPECIFICAZIONE TRASMETTITORIALE}

Tra i molti interessi scientifici di Paolo Mantegazza figurano i suoi lavori sulla funzionalità della giunzione neuromuscolare, le ricerche sul sistema nervoso autonomo periferico e, in particolare, sul ruolo svolto dai neurotrasmettitori nella comunicazione tra neuroni e strutture da loro innervate ed infine sulla possibilità di modulare tali attività attraverso farmaci. Ricordo soprattutto i pioneristici lavori sui curari e sui ganglioplegici che hanno messo le basi per una farmacoterapia allora importante e decisiva per molte patologie $(4,5,6)$.

In questa mia breve lettura vorrei ripercorrere alcune ricerche che abbiamo compiuto assieme al Prof. Mantegazza in un lungo lasso di tempo studiando un tipo particolare di plasticità nel sistema nervoso centrale e periferico indotta da de-nervazione e re-innervazione dei gangli del sistema nervoso autonomo e della muscolatura liscia e striata, plasticità considerata all'inizio "eretica" ma che poi è stata validata da molti dati sperimentali e clinici e che consiste nella possibilità che un neurone maturo possa modificare i suoi neurotrasmettitori attraverso i quali esso comunica con altre cellule, la rispecificazione del neurotrasmettitore, come indicato dal titolo di questa conversazione (7).

Le eccezionali scoperte del primo novecento sulla comunicazione neuronale da parte degli anatomici e biologi cellulari (Santiago Ramon y Cajal e Camillo Golgi) e dei fisiologi (Henry Dale, Adolf Loewi e Charles Sherrington) hanno permesso di stabilire che la maggioranza delle comunicazione tra cellule nervose avviene tramite una speciale struttura, la sinapsi, che permette il passaggio unidirezionale dell'informazione tra due cellule attraverso la liberazione di un neurotrasmettitore dalla cellula presinaptica e il suo riconoscimento da parte della cellula postsinaptica (Fig. 3). Quando ho iniziato a fare ricerca nel settore delle neuroscienze valeva ancora l'assioma di Henry Dale secondo il quale ogni neurone poteva essere identificato in base al neurotrasmettitore che sintetizzava e rilasciava in tutte le sue sinapsi $(8,9)$. Tutto era quindi relativamente chiaro sulle funzioni che un neurone svolgeva in un circuito nervoso: eccitatorie se liberava acetilcolina, glutammato o altri trasmettitori, o inibitorie se liberava GABA o glicina. Ma presto le cose si resero più complesse quando si vide che un neurone oltre al trasmettitore classico poteva rilasciare e co-rilasciare altri trasmettitori di tipo peptidico che potevano modificare le funzioni del neurotrasmettitore classico e più ancora quan- 
do si vide che un neurone poteva sintetizzare e rilasciare più neurotrasmettitori, anche contemporaneamente, per es. durante lo sviluppo, o, nell'animale adulto, in seguito a particolari esigenze funzionali o patologiche, per esempio dopo denervazione-reinnervazione (10).

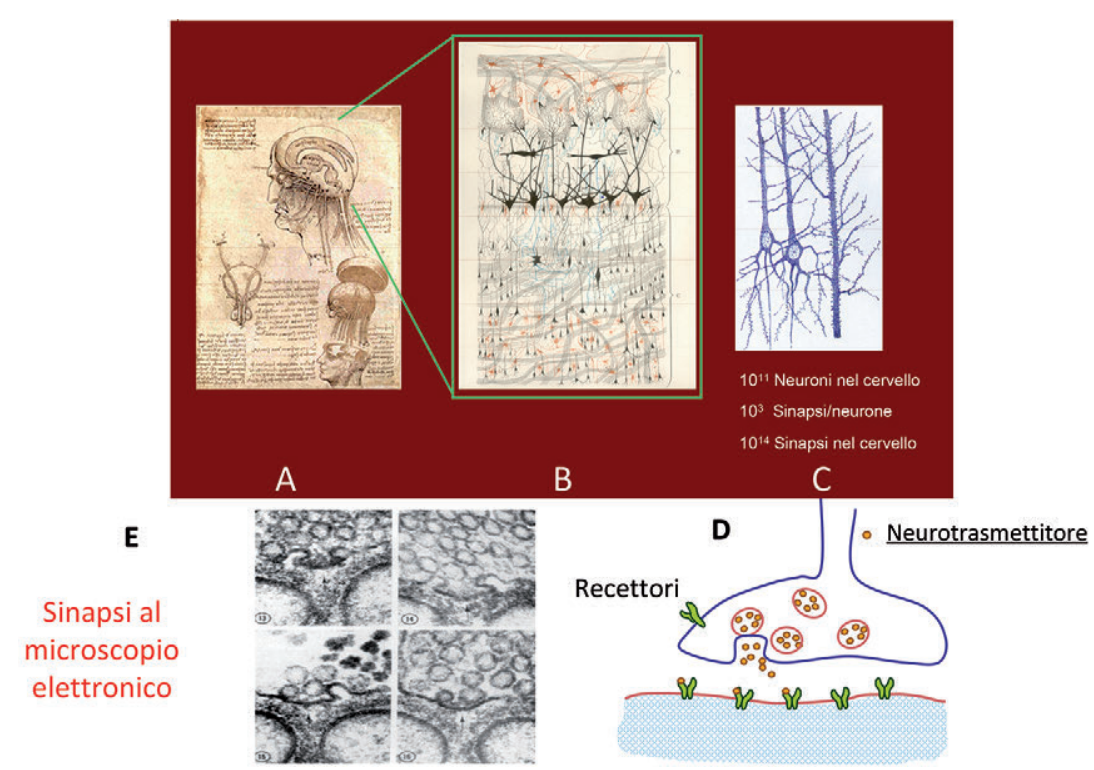

Fig. 3. Schema generale della comunicazione intercellulare nel sistema nervoso centrale. Nel cervello (A), (qui in un disegno di Leonardo) sono presenti circa $10^{11}$ neuroni e un numero leggermente maggiore di cellule gliali. In B una illustrazione di Golgi mostra una sezione del bulbo olfattivo ed esemplifica $i$ numerosi tipi nei quali si differenziano $i$ neuroni e la complessità dei circuiti neuronali che li legano tra loro. La struttura del sistema nervoso è molto precisa, spesso modulare, e fissata nelle sue linee generali dalla conformazione genetica dell'individuo. Lo schema $C$, a più alto ingrandimento, mostra il numero assai elevato di sinapsi, circa $10^{13}$ per neurone e $10^{14}$ in totale. La sinapsi, organulo deputato alla comunicazione diretta tra neuroni, è caratterizzata da un passaggio di informazione unidirezionale, che avviene attraverso la liberazione di neurotrasmettitori, contenuti in vescicole sinaptiche, nello spazio sinaptico dove possono legarsi ai recettori e dare cosi origine al passaggio della informazione (D). L'ingrandimento al microscopio elettronico di una parte dello spazio sinaptico (E) mostra chiaramente il momento nel quale una vescicola sinaptica si apre permettendo l'uscita del neurotrasmettitore che può attivare i recettori posti sulla membrana postsinaptica. Il numero, la forma e la forza delle sinapsi è variabile a seconda delle diverse condizioni funzionali, in modo che la comunicazione sia sempre dinamicamente adattabile e possa rispondere al meglio alle esigenze del momento. La possibilità di modulare questi parametri è alla base della plasticità sinaptica, fenomeno assai complesso nel quale sono coinvolti sia $i$ neuroni che la glia. In questo lavoro descriviamo un tipo particolare di plasticità sinaptica: la rispecificazione trasmettoriale e recettoriale. 
Queste ricerche hanno così aperto un nuovo campo della plasticità sinaptica aggiungendo alla plasticità ottenuta attraverso la modificazione della forma, del numero e della forza delle sinapsi la rispecificazione del neurotrasmettitore $(7,10)$.

Queste ricerche hanno un grande interesse conoscitivo perché consentono di capire meglio le capacità plastiche delle cellule nervose e i rapporti con le strutture innervate, ma anche perché permettono di adottare misure terapeutiche in situazioni gravi di de-nervazione e di sofferenza neuronale periferica. Basti pensare ai traumi stradali, da incidenti sul lavoro e da incidenti durante lo sport che costituiscono una grande calamità in tutti i paesi con cifre che vanno da 40 a 15 eventi per milione di abitanti e che in Italia hanno esitano in paraplegia o tetraplegia nel 40/60\% dei casi (11). Ma, ancora, il problema della re-innervazione è particolarmente importante nei trapianti d'organo perché essa permette una prognosi funzionale molto più positiva dell'organo trapiantato sia esso cuore, o polmone o rene se dopo il trapianto l'organo viene reinnervato $(12,13)$.

\section{LA PLASTICITÀ E LA RISPECIFICAZIONE TRASMETTITORIALE NEL SISTEMA NERVOSO AUTONOMO PERIFERICO}

Le nostre ricerche sulla rispecificazione del neurotrasmettitore sono cominciate all'inizio degli anni 70 del secolo scorso, quando ancora questo fenomeno non era conosciuto, e si sono svolte in due periodi di tempo tra loro molto distanti, ma sempre idealmente collegati tra loro. Il primo gruppo riguarda le capacità rigenerative del sistema nervoso periferico e il secondo gruppo, iniziato negli anni 2004, riguarda invece la possibilità di re-innervare funzionalmente il muscolo striato con fibre non colinergiche.

Per affrontare il primo aspetto abbiamo approntato tre modelli sperimentali in vivo nel gatto e nel coniglio: 1) La re-innervazione del ganglio simpatico cervicale superiore (SCG), denervato, da parte di fibre colinergiche efferenti vagali, mantenendo quindi sempre lo stresso neurotrasmettitore (Acetilcolina), ma inserendo nella regolazione del circuito simpatico una innervazione proveniente dal sistema parasimpatico. 2) La re-innervazione di muscoli effettori periferici come la membrana nittitante $(\mathrm{MN})$ da parte di fibre colinergiche pregangliari dopo asportazione del GCS, sostituendo quindi il neurotrasmettitore (Noradrenalina) che viene normalmente liberato alla periferia con l'acetilcolina. 3) La re-innervazione, in un modello di trapianto in vivo, da 
parte di fibre postgangliari adrenergiche del SCG di iride, che mima una situazione di normale re-innervazione simpatica, e di midollare di surrene che normalmente riceverebbe fibre pregangliari colinergiche. Questi modelli sono illustrati schematicamente nella Fig. 4.

\section{Esperimenti di re-innervazione del sistema nervoso autonomo}
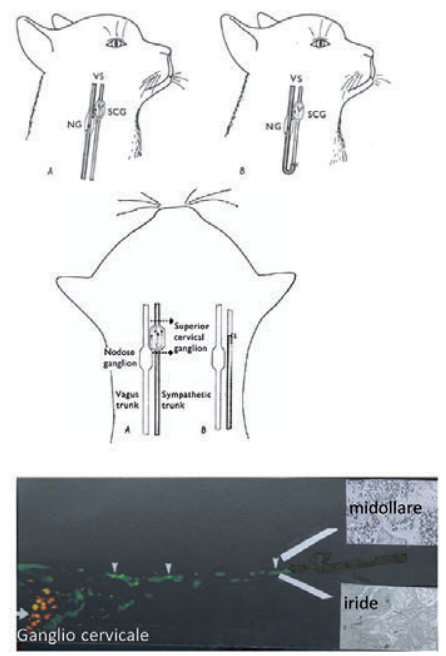

Fibre vagali efferenti poste come presinaptiche nel ganglio cervicale superiore

Fibre pregangliari colinergiche poste come postsinaptiche adrenergiche

Fig. 4. Esposizione schematica degli esperimenti di re-innervazione nel sistema nervoso autonomo per mettere in evidenza la possibile plasticità sinaptica del sistema nervoso periferico sia a livello pre-sinaptico che post-sinaptico. indicati.

Entriamo ora più in dettaglio nei risultati degli esperimenti sopra

Nel primo esperimento abbiamo potuto constatare che un ganglio simpatico poteva essere re-innervato da fibre provenienti dal sistema vagale ottenendo una funzionalità normale della sinapsi gangliare (14). L'esperimento è illustrato nella Fig. 5A, che riporta le MN di animali denervati, a sinistra, e reinnervati a destra, mostra che dopo denervazione vi è mancanza di risposta in vivo della $\mathrm{MN}$ agli stimoli fisiologici e confermata dalla scomparsa delle fibre innervanti (Fig. 5E). Dopo alcuni mesi dall'intervento vi è la completa ripresa della risposta dopo re-innervazione (Fig. 5A,B con le stesse caratteristiche farmacologiche della trasmissione di un ganglio normale (risposta alla nicotina, all'esametonio, alla adrenalina) (Fig. 5B). La microscopia elettronica conferma che la sinapsi gangliare reinnervata (Fig. 5F) è del tutto simile a quella normale (Fig. 5D). 
Fibre vagali efferenti come presinaptiche in ganglio cervicale superiore

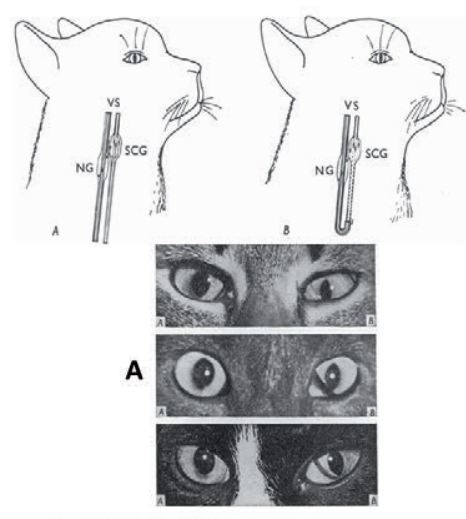

The Journal of Physiology, Vol. 216, No. 1

Plate 3
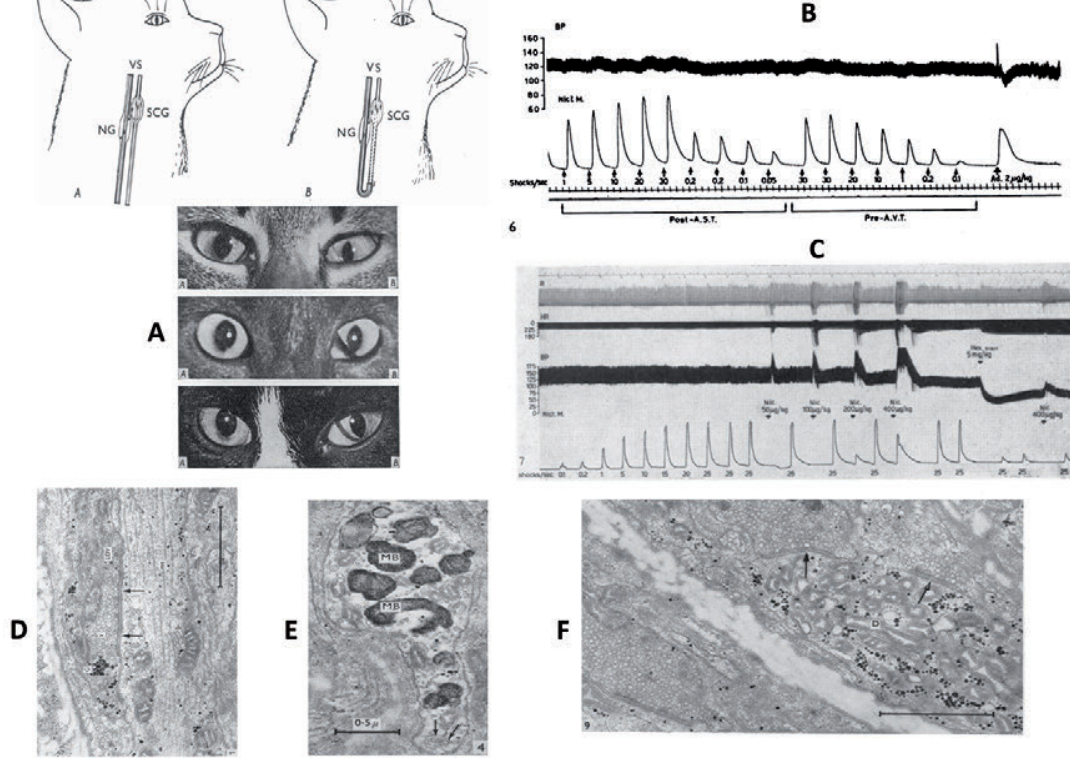

C

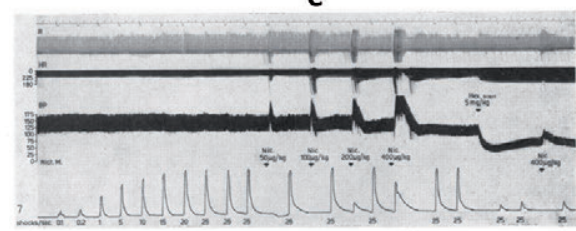

Fig. 5. Esame delle vie nervose rigenerate dopo re-innervazione del GCS da parte di fibre vagali. La re-innervazione della via nervosa è avvenuta in quanto la contrazione della memnrana nittitante $(M N)$ si aveva dopo stimolazioni fisiologiche in vivo $(A$, a sinistra $N M$ dopo denervazione, a destra dopo re-innervazione). B) Registrazione delle contrazioni indotte dalla stimolazione elettrica del tratto pregangliare o post gangliare che mette in evidenza l'avvenuta connessione. Il tracciato superiore registra la pressione arteriosa, mentre quello inferiore le risposte della $M N$ dopo stimolazione del vago. C) I tracciati registrano il respiro, la frequenza cardiaca, la pressione arteriosa e le contrazioni della MN dopo stimolazione pregngliare. La trasmissione gangliare aveva la stessa sensibilità alla nicotina e all'esametonio della sinapsi gangliare normale e la giunzione neuromuscolare periferica continuava ad essere di tipo adrenergico ( $B$, risposta all'adrenalina). La struttura della sinapsi gangliare al microscopio elettronico dopo re-innervazione $(F)$ era del tutto sovrapponibile a quella normale (D) e molto diversa dalla sinapsi denervata (E). (da 14)

La risposta periferica è quindi normale ma si è avuta invece una ristrutturazione dei circuiti nervosi centrali in quanto, in vivo, gli stimoli fisiologici di natura simpatica, per es. retrazione dalla MN provocata da uno stimolo di paura, avvenivano attraverso una eccitazione vagale e non simpatica (14). Si è sviluppata quindi dopo un mese dalla re-innervazione, una plasticità di tutta la risposta comportamentale simpatica che si esplicava attraverso vie centrali e periferiche del tutto nuove. 
Questo punto di grande interesse non è stato allora studiato in modo approfondito in quanto non ne avevamo gli strumenti sperimentali a disposizione per risolvere circuiti centrali così complessi.

Il secondo esperimento ha avuto risultati molto interessanti per la dimostrazione della rispecificazione tramettitoriale. In esso noi abbiamo collegato le fibre pregangliari colinergiche con le fibre posgangliari adrenergiche dopo asportazione del GCS (Figg. 6 e 7). Dopo un lungo periodo di tempo abbiamo trovato che le fibre pregangliari colinergiche innervavano direttamente la muscolatura liscia adrenergica riacquisendo la capacità di sintetizzare e rilasciare catecolamine invece di acetilcolina (15). Le prove funzionali sulla nuova innervazione hanno dimostrato in modo inequivocabile che essa era avvenuta (Fig. 6A), che nel nuovo tronco non vi era presenza di una sinapsi gangliare (Fig. 6B,C) e che la giunzione neuromuscolare periferica aveva conservato le caratteristiche di una giunzione adrenergica (blocco da simpaticolitici) e non rispondeva ai farmaci colinergici (Fig. 6C).

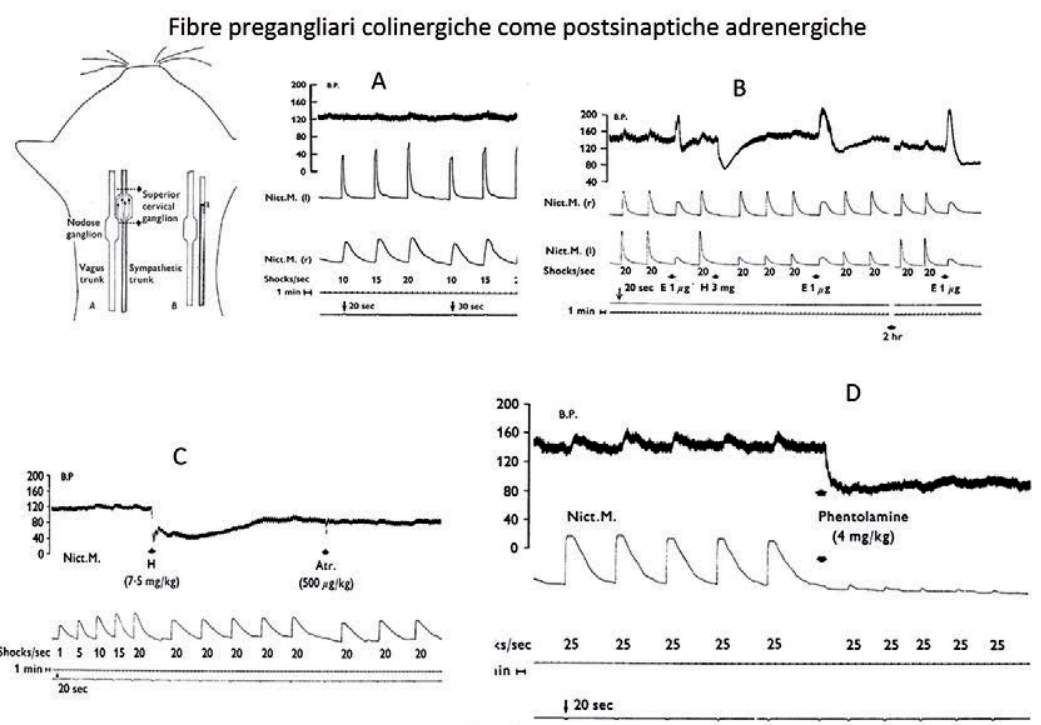

Fig. 6. Esame delle nuove vie nervose dopo asportazione del GCS e anastomosi di fibre pregangliari a fibre postgangliari. I tracciati si riferiscono a pressione arteriosa (superiore) e contrazioni evocate elettricamente della MN (inferiore). La re-innervazione che è avvenuta $(A)$, non contiene una giunzione gangliare in quanto non vi è risposta all' esametonio, che invece è attivo sulla pressione ( $B$ e $C$ ), e la giunzione neuromuscolare continua ad essere adrenergica in quanto risponde alla adrenalina (B) alla fentolamina (D), ma non all'atropina (C). (da 15) 
L'analisi istochimica della $\mathrm{MN}$ ha confermato la presenza di una intensa innervazione adrenergica simile a quella normale (Fig. $7 \mathrm{~A}, \mathrm{~B}) \mathrm{e}$ al microscopio elettronico abbiamo potuto vedere che nelle nuove sinapsi neuromuscolari erano presenti numerose vescicole dense di larghe dimensioni tipiche di terminazioni di tipo adrenergico in formazione (Fig. 7C).
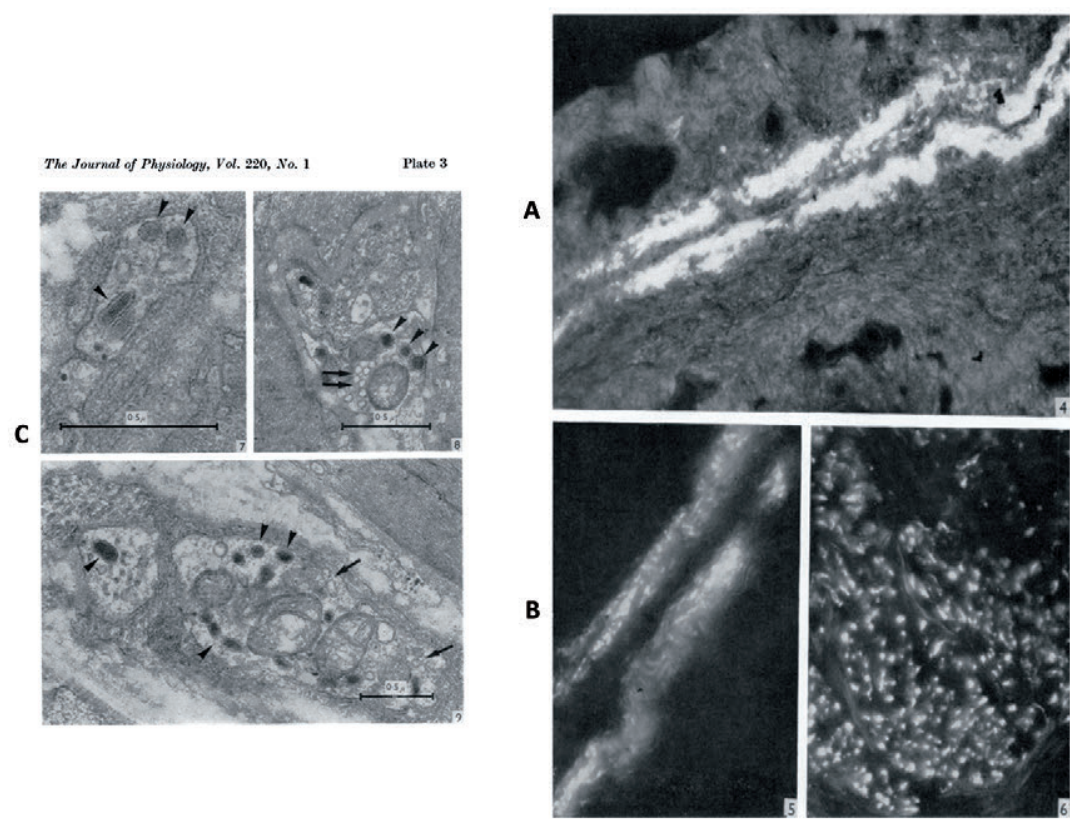

Fig. 7. Ancora un esperimento simile a quello della figura precedente. A-B, Esame istochimico alla formaldeide per la evidenziazione di fibre adrenergiche. In A, a basso ingrandimento, si può vedere una intensa colorazione della tonaca muscolare dell MN normale indice di forte innervazione adrenergica. In $B$, a bassa e più alta risoluzione, la MN dopo reinnervazione da fibre pregangliari, che risulta fortemente innervata da singole fibre adrenergiche. C, la microscopia elettronica permette di vedere all'interno delle nuove terminazioni nervose numerose vescicole dense di largo diametro tipiche di terminazioni adrenergiche in formazione. (da 15)

Questi dati sono di grande interesse perché mostravano per la prima volta che in particolari contesti fisiopatologici le fibre colinergiche pregangliari potevano acquisire o riacquisire la capacità di sintetizzare, immagazzinare e liberare un mediatore adrenergico al posto 
della o assieme alla acetilcolina. Un chiaro esempio di rispecificazione del neurotrasmettitore indotto dalle caratteristiche del bersaglio innervato. I rapporti tra fibra innervante e bersaglio non seguono sempre le stesse leggi apparenti e dipendono probabilmente dalla "forza" reciproca. Un caso nel quale si ha la prevalenza delle fibre innervanti sul bersaglio è quello descritto in letteratura (16) in un esperimento di reinnervazione della $\mathrm{MN}$ da parte del nervo ipoglosso, che naturalmente dovrebbe secernere acetilcolina alle nuove terminazioni. In questo esperimento la nuova innervazione che si sviluppava era, infatti, francamente colinergica con espressione di recettori colinergici nella muscolatura della nittitante e perdita completa di ogni carattere adrenergico (16). E' questo un chiaro esempio di rispecificazione trasmettoriale indotto nel bersaglio e molto diverso da quello da noi descritto nel quale era il bersaglio che dominava sulla fibra innervante. I meccanismi di questa diversa dominanza tra bersaglio e fibra innervante non sono affatto chiari, ma è forse da supporre che siano dipendenti dalla presenza di altre cellule che condizionano i neuroni (17) o dal rilascio di altri fattori, LIF, BDNF,CNTF, da parte della fibra o del bersaglio che possono condizionare l'assetto definitivo (7) oppure dalla attività neuronale stessa, in particolare dalla frequenza, forma e durata degli spikes di $\mathrm{Ca}^{+2}$ intracellulari indotti dai potenziali d'azione spontanei $(18,19)$. E' stato, infatti, dimostrato negli anni seguenti che i neuroni gangliari possono passare da adrenergici a colinergici durante lo sviluppo subendo l'influsso del bersaglio; per esempio le ghiandole sudoripare ricevono all'inizio una innervazione simpatica adrenergica per poi subire durante lo sviluppo postnatale un passaggio rapido da innervazione adrenergica a colinergica, sempre da parte degli stessi neuroni (20). In accordo con queste acquisizioni vi sono anche dimostrazioni che i neuroni gangliari che reinnervano organi bersaglio che esprimono recettori adernergici o colinegici, possono modificare la loro specificità trasmettitoriale passando da adrenergici a colinergici a seconda del bersaglio che devono re-innervare (21) mentre nel sistema motorio il motoneurone ha meno plasticità e detta quindi le condizioni alla periferia. E' possibile che il sistema simpatico sia particolarmente plastico e che possa quindi adattarsi più facilmente da un punto di vista funzionale e molecolare alle esigenze del bersaglio, come suggerito da esperimenti di cultura a lungo temine di neuroni gangliari simpatici (20).

Infine nell'ultimo esperimento che presento abbiamo voluto 
vedere se il SCG, in una situazione che poteva mimare quanto avviene nello sviluppo, era capace di distinguere tra organi che richiedevano una innervazione postsinaptica, iride, da organi che fisiologicamente richiedono una innervazione presinaptica, midollare del surrene (22). Per questo abbiamo trapiantato, in gatti, sotto la capsula renale, spazio molto adatto per far sopravvivere per lungo tempo frammenti di tessuti autologhi, un SCG e a poca distanza un frammento di iride o di midollare del surrene. Dopo un mese abbiamo constatato, attraverso indagini morfologiche, che i tessuti trapiantati erano ben sopravvissuti e che il GCS aveva innervato molto bene l'iride ma che nessuna fibra postsinaptica aveva raggiunto la midollare del surrene (Fig. 8). I rapporti, quindi tra neurone innervante e bersaglio, che abbiamo visto prima così plastici in esperimenti in vivo nei quali si conserva tutta la complessità di sistemi innervanti ed innervati, se ricondotti ai suoi elementi essenziali in qualche modo isolati perdono di plasticità e si rifanno ad uno schema più "primitivo" nel quale la gerarchia tra organo innervante ed innervato è ben precisa e codificata.

Nel complesso i risultati di questi tre esperimenti dimostrano la grande plasticità di risposta del sistema nervoso periferico adulto in vivo a situazioni non fisiologiche che riesce, come nel primo esperimento, a mantenere una struttura periferica funzionante ma con un sconvolgimento e riconversione dei circuiti neuronali centrali; a modificare le proprietà biologiche di neuroni, modificando la loro specificazione trasmettitoriale, come nel caso del secondo esperimento; ma continua a mantenere regole fondamentali di gerarchia tra neuroni innervanti e cellule innervate in situazioni di trapianti sperimentali isolati da ogni contesto complesso di circuiti nervosi, dimostrando la capacità delle cellule innervate di richiamare o respingere determinate fibre nervose.

Questi dati, anche se non sono di facile interpretazione per quanto riguarda i meccanismi molecolari e cellulari con i quali si attuano, danno però una chiara dimostrazione che in alcuni casi esiste la possibilità, anche nell'animale adulto, di modificare la specificazione del neurotrasmettitore, e che il tessuto autonomo periferico ha una grande plasticità per ristabilire le sue funzioni anche in condizioni strutturali non fisiologiche. Questi dati possono fornire delle indicazioni su possibili interventi di re-innervazione anche non canonici nei trapianti d'organo e nella re-innervazione chirurgica post traumatica del sistema nervoso periferico. 
Fibre gangliari postsinaptiche come postgangliari (iride) o pregangliari (midollare)

A

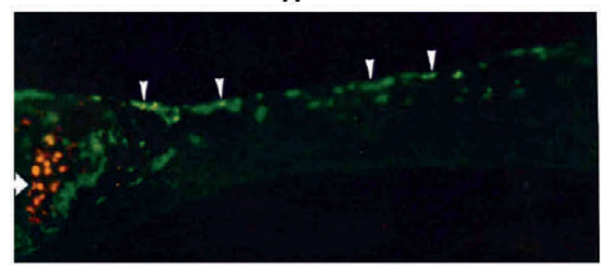

C

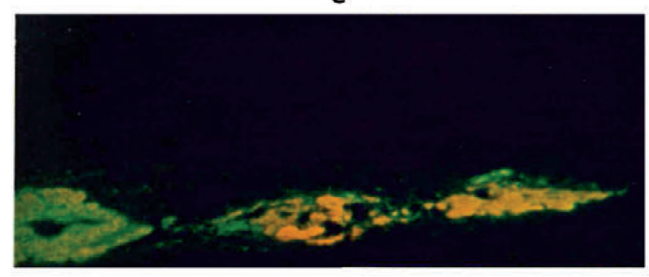

B

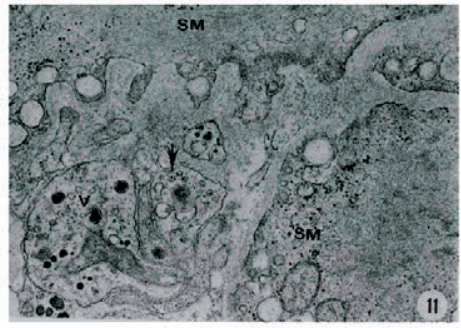

D

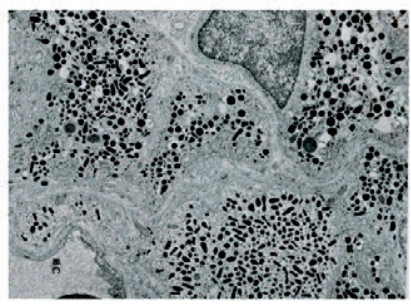

Fig. 8. Co-trapianto di GCS con iride (A, B) o con midollare del surrene (C, D). In A si può osservare il GCS che contiene numerosi neuroni gangliari (punti arancione) e numerose fibre adrenergiche che raggiungono l'iride (teste di freccia); in B è mostrata l'iride trapiantata esaminata al microscopio elettronico che permette di evidenziare delle tipiche terminazioni adrenergiche, ricche di vescicole dense, nella tonaca muscolare dell'iride. E' interessante notare l'aspetto molto simile tra queste terminazioni e quelle raffigurate nella Fig. 7C. In C è illustrata la situazione del GCS (struttura a sinistra) trapiantato vicino a due frammenti di midollare del surrene; l'intensa colorazione gialla indica il forte contenuto in catecolamine delle cellule midoollari. Si può osservare che non ostante la grande vicinanza dei due tessuti non vi sia passaggio di fibre tra il GCS e la midollare. L'analisi della midollare al microscopio elettronico (D) conferma la mancanza di fibre innervanti. (da 22)

\section{LA PLASTICITÀ E LA RISPECIFICAZIONE RECETTORIALE NELLA GIUNZIONE NEUROMUSCOLARE STRIATA}

Non abbiamo potuto continuare questi esperimenti sul sistema autonomo periferico soprattutto per la difficoltà di poter eseguire su animali idonei questi interventi, pur rimanendo vivo l'interesse nella nostra mente di ricercatori. L'occasione di riprendere questa linea di ricerca ci è stata offerta, più di trenta anni dopo, dal gruppo del prof. Brunelli di Brescia che, assieme ai colleghi farmacologi, aveva evidenziato la possi- 
bilità di re-innervare in modo funzionale, nel ratto, muscoli striati denervati con fibre nervose che derivavano dal nucleo rosso e dalla formazione reticolare del complesso vestibolare (23). L'interesse di fondo consisteva, ancora una volta, nel fatto che le fibre innervanti erano di tipo glutammatergico e non colinergico come quelle dei normali motoneuroni, che la trasmissione neuromuscolare si tramutava da colinergica nicotinica in glutammatergica (Fig. 9) e che il muscolo re-innervato assumeva una funzionalità molto simile a quella fisiologica (23). Ci sembrava quindi che fossimo in presenza di un nuovo tipo di plasticità dovuta ad una rispecificazione, questa volta non del neurotrasmettitore ma del recettore nel tessuto re-innervato, e nello stesso tempo, ancora una volta, ad una rielaborazione dei circuiti cerebrali centrali.

In questa intensa e fruttuosa collaborazione alla quale hanno preso parte farmacologhi, fisiologi, morfologi, esperti di trasmissione neuromuscolare e chirurghi, abbiamo potuto riconfermare, attraverso la immunolocalizzazione al microscopio ottico ed elettronico, che le fibre centrali glutamatergiche di origine dal nucleo rosso re-innervano il muscolo seguendo il nuovo percorso sperimentale senza modificare il neurotrasmettiotore originario (Fig. 9), che le nuove terminazioni re-innervavano le fibre muscolari negli stessi siti prima innervati dalle fibre colinergiche (Figg. 10 e 11); che le nuove fibre re-innervanti sintetizzavano e immagazzinavano nelle vescicole sinaptiche glutammato (Fig. 11); che il 30\% delle cellule muscolari re-innervate esprimevano ex novo recettori per il glutammato di tipo GluR1; ed infine che nelle giunzioni re-innervate erano presenti sia recettori glutamatergici che recettori nicotinici (Fig. 10) (24).

Ancora una volta eravamo in presenza di un fenomeno straordinario di plasticità sinaptica in un sistema periferico ma con forti ripercussioni sui circuiti del sistema nervoso centrale. Da una parte, le cellule muscolari, innervate da fibre glutamatergiche, esprimevano ex novo tutto il macchinario enzimatico e molecolare per la produzione, il trasporto e l'inserimento corretto nella giunzione dei recettori glutamatergici facendo risaltare quanto sia plastico il sistema muscolare e sensibile a profondi cambiamenti a seconda dell'attività e della neurochimica delle fibre innervanti. Si trattava quindi veramente di una nuova forma di plasticità: una respecificazione recettoriale che non avevamo visto negli esperimenti sul sistema simpatico. Dall'altra parte i neuroni del complesso vestibolare, che non controllano fisiologicamente la funzionalità muscolare, dopo la re-innervazione eterologa, inducevano nella muscolatura re-innervata efficacemente una funzionalità molto simile a quella fisiologica. 


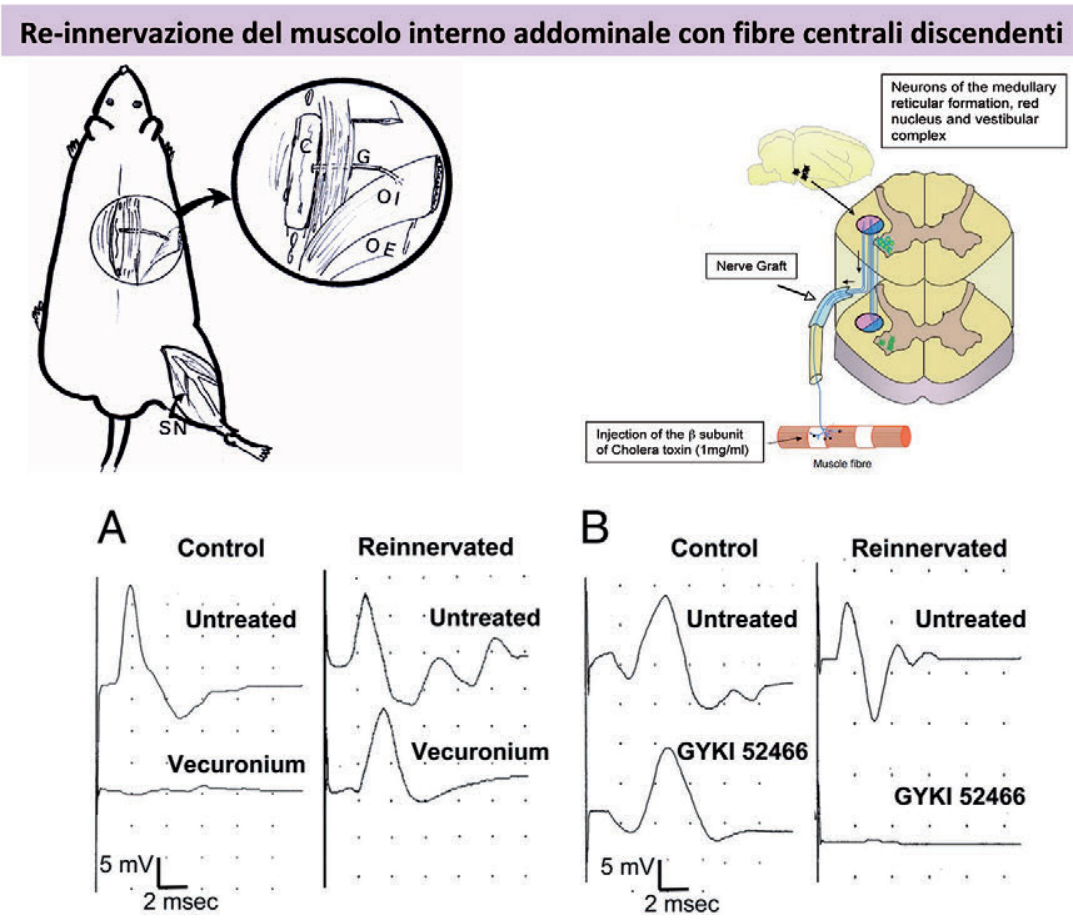

Fig. 9. Re-innervazione del muscolo obliquo addominale di ratto denervato da parte di fibre glutamatergiche discendenti del midollo, e provenienti dal nucleo rosso, convogliate al muscolo da un troncone nervoso prelevato dal nervo sciatico. Nella parte superiore si vede lo schema dell'intervento chirurgico e lo schema della via re-innervante. Si è potuto risalire all'origine delle fibre innervanti iniettando nel muscolo re-innervato della tossina colerica marcata e seguendola retroattivamente fino ai nuclei centrali. Nella parte in basso si può osservare un tipico tracciato elettromiografico ottenuto stimolando il tronco nervoso innervante il muscolo che indica chiaramente che: 1- l'innervazione muscolare è avvenuta attraverso il nuovo tronco nervoso sperimentale ma che la trasmissione neuromuscolare non è colinergica in quanto non è sensibile al curaro come nel muscolo normale (A); 2- la nuova innervazione neuromuscolare è invece abolita dal GYKI 52466, un bloccante dei recettori glutamatergici AMPA GluR1 e GluR2; 3- che l'inibitore glutamatergico non ba effetto sulla trasmissione neuromuscolare normale. (da 23)

Noi abbiamo chiaramente documentato, anche con molti dettagli molecolari che non ho qui riportato, che la rispecificazione recettoriale nelle nostre condizioni sperimentali è avvenuta, ma non sono noti i meccanismi per i quali essa si attua. 


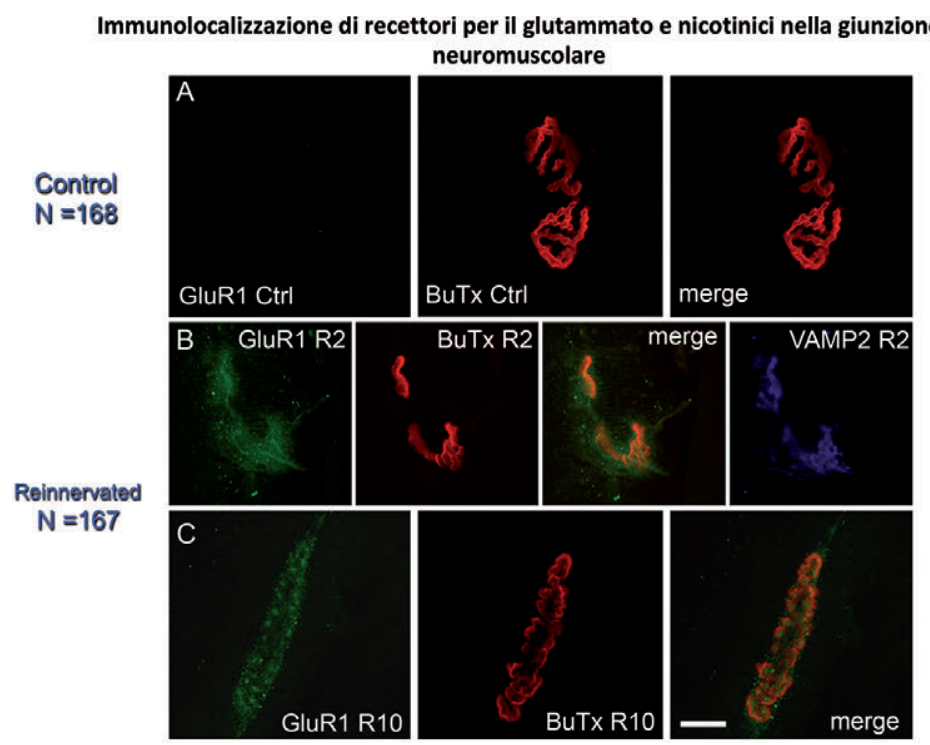

Fig. 10. Ancora esperimento della figura precedente. Visualizzazione di marcatori per $i$ recettori glutamatergici GluR1 e per i recettori nicotinici (BuTx) in giunzioni neuromuscolari di muscoli normali (A) e re-innervati dopo 2 (B) e 10 (C) mesi dall'intervento. Si può osservare che nel muscolo normale non vi è traccia di recettori glutamatergici mentre è intensa la presenza di ricettori nicotinici $(A)$. Nel muscolo re-innervato $(B, C)$ già dopo 2 mesi dall'intervento sono presenti ambedue $i$ tipi recettoriali che si localizzano nelle stesse giunzioni neuromuscolari in circa il 10\% delle fibre. La presenza nelle giunzioni di VAMP2 indica che la re-innervazione è avvenuta. (da 24).

Glutammato, i suoi trasportatori e i recettori glutamatergici sono presenti in alcuni muscoli striati, come quelli dello sfintere esofageo di alcuni mammiferi e in alcuni muscoli striati degli invertebrati $(25,26)$, ma gli esperimenti finora riportati non potevano evidenziare con chiarezza la presenza del trasmettitore nella fibra presinaptica e nemmeno la localizzazione dei recettori per il glutammato a livello post sinaptico (27); inoltre, gli studi sulla funzione del sistema glutamatergico sulla giunzione neuromuscolare della rana (28) e del ratto (29) e in alcune zone del sistema nervoso durante lo sviluppo (30) sembrano dimostrare che l'apparato glutamatergico presente in quelle giunzioni sia rivolto soprattutto alla modulazione della efficienza e della forza di trasmissione della sinapsi colinergica, modulando la liberazione di acetilcolina dalla presinapsi, un tipico evento modulatorio da parte di un neurotrasmettitore co-liberato con l'acetilcolina (10). 
A

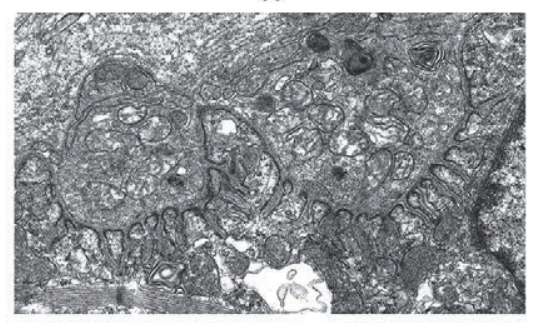

B

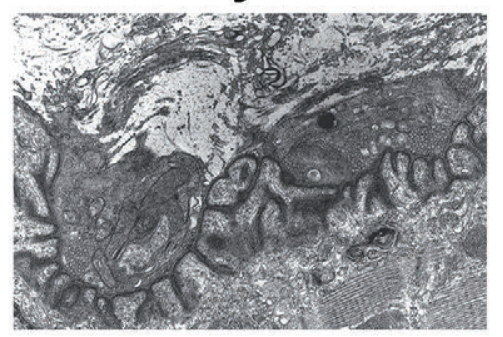

C

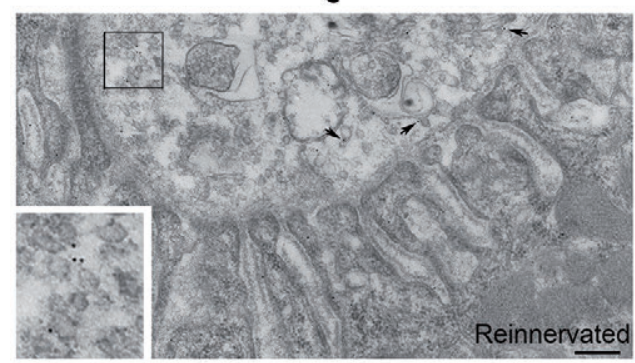

D

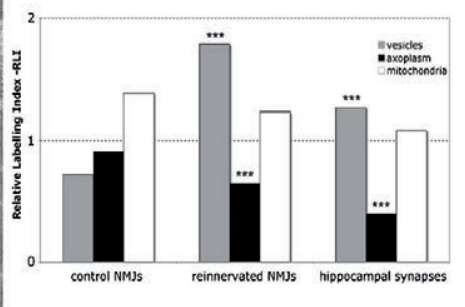

Fig. 11. Presenza e localizzazione del glutamato nelle nuove giunzioni neuromuscolari. La figura mostra che l'aspetto morfologico delle giunzioni neuromuscolari contenenti le fibre reinnervanti (B) è del tutto sovrapponibile a quello delle giunzioni normali (A). In $C$ è dato un esempio di localizzazione nella giunzione neuromuscolare re-innervata delle particelle d'oro che marcano le molecole di glutamato e si può notare la loro presenza nelle vescicole sinaptiche. In D è riportata l'analisi statistica della immuno-localizzazione del glutammato nelle giunzioni neuromuscolari di muscolo normale, di muscolo re-innervato e nell' ippocampo, tipica zona assai ricca di terminazioni glutamatergiche. L'analisi dimostra che mentre nel muscolo normale la distribuzione delle particelle d'oro è casuale e non differente dal background, nella giunzione neuro muscolare re-innervata le particelle d'oro sono prevalentemente nelle vescicole sinaptiche come nelle sinapsi glutamatergiche dell'ippocampo. (da 24)

Di fronte ad una ipotizzata, ma non del tutto dimostrata, presenza di recettori glutamatergici postsinaptici nella giunzione neuromuscolare di mammiferi adulti, noi abbiamo documentato che essi non sono mai presenti nel muscolo obliquo interno addominale di ratto esaminati a livello ultrastrutturale, immuno-chimico e molecolare. Basandosi sui nostri dati e su quelli della letteratura è possibile ipotizzare almeno due meccanismi con i quali si manifesta l'espressione di questa nuova componente recettoriale. L'ipotesi più semplice, basata sulla interpretazione di alcuni dati che abbiamo citato, è che ogni muscolo striato possegga 
già efficiente, anche nello stato adulto, la via molecolare per la produzione, inserzione e regolazione dei recettori glutamaterici ma che essa sia inibita attraverso stimoli fisiologici (corretta attività presinatica, frequenza e grado di stimolazione, liberazione di co-trasmettitori) e che il nostro intervento sperimentale attraverso una forte stimolazione da parte delle nuove terminazioni glutamatergiche possa avere riattivato questa via "dormiente" aumentando il numero di recettori glutamatergici già presenti ma in numero non rilevabile dalle nostre tecnologie anche le più sensibili. La seconda ipotesi, forse più probabile, è che $\mathrm{i}$ recettori glutamatergici siano veramente assenti dalla parte postsinapica della giunzione neuromuscolare, ma che la nuova stimolazione glutamatergica abbia potuto riattivare lo sviluppo della via genetica che porta alla espressione del macchinario recettoriale glutamatergico magari attraverso la presenza di altri trasmettitori/modulatori o fattori di crescita liberati dalle terminazioni glutamatergiche. Convergono con questa ipotesi i lavori che illustrano che nella giunzione neuromuscolare di tipo colinergico nicotinico le terminazioni rilasciano, oltre che l'acetilcolina, anche il fattore di crescita e differenziamento ARIA e che esso regola il numero e la dinamica dei recettori nicotinici postsinaptici (31), o quelli nei quali si mostra che lo stesso fattore possa indurre in neuroni cerebellari in cultura l'espressione di recettori per il glutammato di tipo NMDA (32).

\section{CONCLUSIONI}

Queste esperienze, assieme a molte altre ormai presenti in letteratura (7), dimostrano che nel sistema nervoso periferico e neuromuscolare la formazione di sinapsi efficienti, funzionali e affidabili dipende dalla interazione dinamica e coordinata di molte molecole presenti sia nella parte neuronale innervante sia nel bersaglio innervato. Mimando quanto avviene nello sviluppo, si è visto che è possibile modificare e modulare questa interazione dinamica e attivare una plasticità sinaptica, basata su una diversa specificazione dei neurotrasmettitori e dei loro recettori, attraverso interventi sperimentali e dare in questo modo origine a circuiti nervosi non canonici ma ugualmente funzionali. Si è così osservato che il sistema nervoso periferico adulto, sia autonomo che muscoloscheletrico, ha una plasticità prima impensabile sia a livello delle strutture periferiche sia a livello dei circuiti complessi cerebrali che lo governano. In 
tutti gli esperimenti mostrati, sia quello sul sistema simpatico che quello sul sistema muscolare, l'intervento chirurgico periferico ha pesantemente coinvolto i circuiti centrali di controllo: nel caso del simpatico si è osservato che la regolazione della risposta del GCS, simpatico, reinnervato dal vago, avveniva correttamente attraverso l'attivazione dei nuclei del vago, che normalmente agiscono in alternativa alla stimolazione simpatica; nel caso del muscolo obliquo re-innervato da fibre glutamatergiche del Nucleo Rosso del complesso vestibolare si osservava che il muscolo in vivo aveva delle risposte funzionali abbastanza simili a quelle fisiologiche guidate da questo complesso che normalmente non ha niente a che fare con la regolazione del movimento muscolare. Analizzare in dettaglio questi fenomeni nei nostri modelli sperimentali è troppo complesso, anche per il numero degli animali assai limitato e la nostra competenza impreparata a tale scopo. Purtuttavia abbiamo potuto mettere in evidenza che questi complessi e plastici rapporti tra sistema nervoso centrale e periferico possono essere manipolati ed utilizzati anche per correggere situazioni patologiche.

Questi dati possono aprire un campo ancora poco esplorato di grande interesse biologico per capire come venga regolata la specificità neuronale ma anche traslazionale perché permette di immaginare esperimenti di re-innervazione non canonici ma forse ugualmente funzionali.

\section{RINGRAZIAMENTI}

Questa piccola review è frutto di una collaborazione molto intensa e fattiva con gli autori di molte delle ricerche riportate e che voglio qui ricordare con molto affetto e stima: Paolo Mantegazza e Bruno Ceccarelli, che tanto hanno mi dato nel lungo tempo speso assieme nella ricerca e che oggi purtroppo non possono più qui godere con noi di questi interventi, e Giorgio Brunelli, Sergio Barlati, Mario Buffelli, Michele Carruba, Maura Francolini, Marina Pizzi e Pier Franco Spano, che hanno contribuito con la loro alta professionalità, costanza e originalità allo sviluppo di queste innovative linee di ricerca. 


\section{BIBLIOGRAFIA}

1. Berti F, Chiesara E, Clementi F, Montorsi W, Sironi W. Uomini e farmaci. La farmacologia a Milano tra storia e memoria, Laterza Editori, Bari, 2002.

2. Schmid SL and Farquhar MG. The Palade Symposium: Celebrating Cell Biology at Its Best. Molecular Biology of the Cell 2010, 21, 2367-2370.

3. Greengard P. The Neurobiology of Dopamine Signaling, in Nobel lectures, Physiology or Medicine 1996-2000, Word Scientific Publishing, Singapore, 2003.

4. Cavallini G, Mantegazza P, Massarani E, Tommasini R. The ganglion-blocking activities of some alkylamine derivatives of stilbene and of biphenyl. Farmaco Sci. $1953,8,317-31$.

5. Mantegazza P. Curare-like properties of a new series of symmetrical quaternary ammonium salts: 2, 2'-polymethylene-bis-piperidinium and pyridinium compounds. Farmaco Sci. 1956, 11:357-77.

6. Barzaghi F, Mantegazza P, Riva M. Effects of some guanidine derivatives on neuromuscular and ganglionic transmission. Br J Pharmacol Chemother., 1962, 19, 414-26.

7. Spitzer N. Neurotransmitter switching? No surprise. Neuron 2015, 86, 1131-44.

8. Eccles JC, Fatt P, Koketsu K. Cholinergic and inhibitory synapses in a pathway from motor-axon collaterals to motoneurones, J Physiol (Lond), 1954, 126: 524-62.

9. Strata P, Harvey R. Dale's principle, Brain Res Bull. 1999, 50, 349-50.

10. Hnasko TS, Edwards RH. Neurotransmitter Corelease: Mechansm and Physiological Role., Annu. Rev. Physiol., 2012, 74, 225-43.

11. Singh A, Tetrault L, Kalsi-Ryan S, Nouri A, Fehlings MG. Global prevalence and incidence of traumatic spinal cord injury, Clinical Epidemiology, 2014, 6, 309- 331.

12. Bengel FM, Ueberfuhr P, Schiepel N, Nekolla SG, Reichart B, Schwaiger M. Effect of Sympathetic reinnervation on cardiac performance after heart transplantation, N Engl J Med, 2001, 345, 731.

13. Shioshvili T. Surgical reinnervation of the transplanted kidney: is it needed? Eur Urol. 1988, 14, 56-60.

14. Ceccarelli B, Clementi F, Mantegazza P. Synaptic transmission in the superior cervical ganglion of the cat after re-innervation by vagus fibres, J Physiol, 1971, 216, 87-98.

15. Ceccarelli B, Clementi F, Mantegazza P. Adrenergic re-innervation of smooth muscle of nictitating membrane by preganglionic sympathetic fibres, J Physiol, 1972, 220, 211.

16. Koslow SH, Giacobini E, Kerpel-Fronius S, Olson L. Cholinergic Transmission in the hypoglossal reinnervated nictitating membrane of the cat: an enzymtic, histochemical and physiological study. J Pharmac Exp Therap, 1972, 180, 668-671.

17. Patterson PH, Chun L. The influemce of non-neuronal Cells on catecholamine andacetylcholine sunthesis and accumulation in culture of dissociated sympathetic neurons. Proc Natl Acad Sci ,USA, 1974, 71, 3607-10.

18. Spitzer NC. Activity dependent neutransmitter respecification, Nature Rev Neurosci , 2012, 13, 94-106. 
19. Walicke PA, Campenot RB, Patterson PH. Determination of transmitter function by neuronal activity, Proc Natl Acad Sci USA, 1977, 74, 5767-71.

20. Schotzinger RJ, Landis SC. Cholinergic Phenotype developed by noradrenergic sympathetic neurons after innervation of a novel cholinergic target in vivo. Nature, 1988, 335, 637-639.

21. Wakshull E, Johnson MI, Burton H. Postnatal rat sympathetic neurons in culture. 1l. Synaptic transmission by postnatal neurons. J Neurpophysiol 1979, 42; 1426-42.

22. Carruba M, Ceccarelli B, Clementi F, Mantegazza P. Selectivity in the reinnervation of the iris and adrenal medulla by superior crvical ganglion after transplantation under the kidney capsule. Brain Res 1974, 77, 39-53.

23. Brunelli G, Spano PF, Barlati S, Guanieri B, Barbon A, Bresciani R, Pizzi M. Glutamatergic reinnervation through peripheral nerve graft dictates assembly of glutamatergic synapses at rat skeletal muscle. Proc Natl Acad Sci USA, 2005, 202, 8752-57.

24. Francolini M, Brunelli G, Cambianica I, Barlati S, Barbon A, La Via L, Guanieri B, Boroni F, Lanzillotta A, Baiguera C, Ettorre M, Buffelli M, Spano PF, Clementi F, Pizzi M. Glutamatergic reinnervation and assemmbly of glutamatergic synapses in adult rat skeletal muscle occurs at cholinergic endplates. J Neuropath Exp Neurol, 2009, 68, 1103-1115.

25. Petersen SA, Fetter RD, Noordermeer JN, Goodman CS, DiAntonio A. Genetic analysis of glutamate receptors in Drosophila reveals a retrograde signal regulating presynaptic transmitter release, Neuron. 1997, 19, 1237-48.

26. Fox LE, Lloyd PE. Glutamate is a fast excitatory transmitter at some buccal neuromuscular synapses in Aplysia. Neurophysiol. 1999, 82,1477-88.

27. Mays TA, Sanford JL, Hanada T, Chishti AH, Rafael-Fortney JA. Glutamate receptors localize postsynaptically at neuromuscular junctions in mice. Muscle \& Nerve, 2009, 39, 343-9.

28. Pinard A, Lévesque S, Vallée J, Robitaille R. Glutamatergic modulation of synaptic plasticity at a PNS vertebrate cholinergic synapse, Eur J Neurosci. 2003,18, 3241-50.

29. Waerhaug O, Ottersen OP. Demonstration of glutamate-like immunoreactivity at rat neuromuscular junctions by quantitative electron microscopic immunocytochemistry. Anat Embryol (Berl); 1993,188, 501-13.

30. Boulland JL, Qureshi T, Seal RP, Rafiki A, Gundersen V, Bergersen LH, Fremeau RT Jr, Edwards RH, Storm-Mathisen J, Chaudhry FA. Expression of the vesicular glutamate transporters during development indicates the widespread corelease of multiple neurotransmitters. J Comp Neurol., 2004, 480, 264-80.

31. Fischbach GD, Rosen KM. ARIA: a neuromuscular junction neuregulin. Annu Rev Neurosci. 1997, 20, 429-58.

32. Ozaki M, Sasner M, Yano R, Lu HS, Buonanno A. Neuregulin-beta induces expression of an NMDA-receptor subunit. Nature. 1997, 390, 691-4. 